\title{
Sentiment d'injustice et engagement. Les expressions militantes de chocs moraux en contexte coercitif ou autoritaire
}

Feelings of Injustice and Political Engagement: Activist Expressions of Moral

Shock in Coercive or Authoritarian Contexts

Pénélope Larzillière

\section{(2) OpenEdition}

12 Journals

\section{Édition électronique}

URL : https://journals.openedition.org/conflits/20382

DOI : $10.4000 /$ conflits. 20382

ISSN : $1777-5345$

Éditeur :

CECLS - Centre d'études sur les conflits - Liberté et sécurité, L'Harmattan

\section{Édition imprimée}

Date de publication : 10 octobre 2018

Pagination : 157-177

ISBN : 978-2-343-16812-8

ISSN : 1157-996X

Référence électronique

Pénélope Larzillière, «Sentiment d'injustice et engagement. Les expressions militantes de chocs moraux en contexte coercitif ou autoritaire », Cultures \& Conflits [En ligne], 110-111 | été/automne 2018, mis en ligne le 10 octobre 2020, consulté le 07 janvier 2022. URL : http://

journals.openedition.org/conflits/20382 ; DOI : https://doi.org/10.4000/conflits.20382 


\section{Sentiment d'injustice et engagement. Les expressions militantes de chocs moraux en contexte coercitif ou autoritaire}

\section{Pénélope LARZILLIERE}

Pénélope Larzillière est sociologue, habilitée à diriger des recherches, chargée de recherche à l'Institut de Recherche pour le Développement (IRD), rattachée au CEPED (Université Paris Descartes-IRD). Ses travaux portent sur l'engagement politique et le militantisme, y compris les formes extrêmes, et sur les circulations idéologiques. Elle travaille également sur l'art contestataire. Parmi ses publications, on compte notamment les ouvrages Activism in Jordan (Zed Books, 2016), La Jordanie contestataire. Militants islamistes, nationalistes et communistes (Actes Sud, 2013) et Être jeune en Palestine (Balland, 2004). Elle a également co-dirigé les numéros de revue "Révolutions, contestations, indignations " (Socio, $n^{\circ} 2,2013$ ), et " Faut-il désoccidentaliser l'bumanitaire?" (Humanitaire, $n^{\circ}$ 24, 2010).

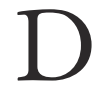
ifférents registres émotionnels existent et coexistent autour des mêmes causes : colère, amertume, admiration, enthousiasme, la liste est longue. Parmi ces registres émotionnels, celui des sentiments moraux est spécifique, en ce qu'il combine dans son expression affectivité et références axiologiques, et se caractérise ainsi par une certaine stabilité, ou durabilité. Le sentiment d'injustice en fait partie et nous voudrions revenir ici, dans une perspective sociologique, sur la constitution de l'émotion comme expérience morale de l'injustice dans le rapport à l'engagement. Qu'en est-il de ces rapports entre sentiments d'injustice et engagement dans les contextes coercitifs ou autoritaires? C'est ce que nous voudrions interroger spécifiquement.

L'idée d'un choc moral conduisant à l'engagement a été mise en avant en sociologie des émotions politiques. Ainsi James Jasper, pour accompagner sa réflexion sur le rôle des émotions dans les mouvements sociaux, utilise la notion de choc moral à définir pour lui comme un malaise [unease] viscéral suite à certains événements ou informations. Un malaise conduisant à une 
prise de distance, une réévaluation du monde et une remise en cause ${ }^{1}$. Il peut s'appuyer sur les récits de militants qui, bien souvent, reviennent sur une première expérience, à la source d'une prise de conscience de l'injustice et de la volonté de lutter contre. William Gamson définit le cadre de l'injustice comme une indignation morale où conscience politique et émotion se mêlent étroitement ${ }^{2}$. Et si l'on peut aller au-delà d'une analyse en termes de cadres au profit d'une approche plus processuelle et dynamique, il reste que le sentiment d'injustice présente la caractéristique de tresser éléments idéels, référence à des valeurs et ancrage émotionnel. Cette combinaison qui permet d'articuler le domaine affectif aux mises en sens développées explique que ce sentiment soit spécifiquement mis en avant dans l'engagement.

Les analyses portant sur l'impact d'un « choc moral» ou sur les cadres de l'injustice ont d'abord été élaborées à partir de certains contextes occidentaux démocratiques actuels ${ }^{3}$; si répression et coercition peuvent y exister, elles n'atteignent ni la systématicité ni la violence des contextes coercitifs et autoritaires. Nous voudrions, alors, opérer ici un certain « décentrement », en nous concentrant sur ce type de contextes, non pour conclure à l'exceptionnalisme, mais pour interroger la façon dont l'expérience de la violence politique peut être prise en compte dans l'analyse de l'expression d'un sentiment d'injustice en lien avec un engagement. Les expériences de coercition politiques n'induisent pas toujours les mêmes réactions et sont loin de conduire nécessairement à un engagement, voire l'inhibent, ce qui représente d'ailleurs un des objectifs de la répression ${ }^{4}$. Nous formulons, ainsi, l'hypothèse que cette expérience spécifique de la violence politique se traduit par une intensification émotionnelle dont l'impact est à considérer, sans tomber, ni dans une analyse qui lierait en de fausses évidences le choc induit par certaines expériences, le sentiment d'injustice et l'engagement, ni à l'inverse dans une vision aseptisée et stratégique des militants ${ }^{5}$.

1. Jasper J., "Constructing Indignation: Anger Dynamics in Protest Movements ", Emotion Review, vol. 6, $\mathrm{n}^{\circ}$ 3, juillet 2014, p. 210, et pour de plus amples développements : Jasper J., The Art of Moral Protest, Chicago, University of Chicago Press, 1997.

2. GamsonW. A., Talking Politics, Cambridge University Press, 1992, p. 7.

3. Dans The Art of Moral Protest, James Jasper s'appuie avant tout sur les mobilisations aux États-Unis (en particulier les mouvements antinucléaires et pour les droits des animaux), même s'il élargit aussi au XIXe siècle et à l'Irlande avec l'exemple de la Land League. Le chapitre qui porte sur les Khmers rouges ne traite pas du choc moral en tant que tel, mais de la façon dont des organisations intègrent le cadre de l'injustice dans des idéologies totales et abstraites qui essentialisent l'ennemi et les conséquences destructrices qui en découlent. William Gamson s'appuie sur l'analyse de discussions politiques au sein de groupes de « working people » aux États-Unis, autour de quatre thèmes médiatisés, et objets de controverses.

4. Sur la variabilité des effets de la répression sur le militantisme, voir le dossier : Cuadros D. et D. Rocha (dir.), « Militantisme et répression », Cultures E Conflits, n 89, printemps 2013.

5. Alice Verstraeten montre ainsi dans le cas des familles de disparus en Argentine, qui s'engagent contre l'impunité à partir de 1977, combien il est nécessaire de prendre en compte le caractère traumatisant et exceptionnel de leur expérience et leurs émotions pour comprendre les «tactiques » qu'elles mettent en place. Verstraeten A., "L'engagement “en réseau" contre l'impunité : des familles de "disparus" défient le "no te metas" ", Tracés. Revue de Sciences humaines [En ligne] URL : http://journals.openedition.org/traces/236, novembre 2006. 
Car, et nombre de commentateurs de la théorie du choc moral de James Jasper l'ont noté 6 , cette approche soulève deux questions. La première concerne le caractère intrinsèquement choquant de certaines scènes et expériences. Que les expériences de brutalité, de violence, d'oppression, certaines scènes et certains vécus, suscitent nécessairement un profond sentiment d'injustice et révolte pourrait relever de l'évidence (peut-être le souhaiterait-on). Mais il apparaît assez rapidement que, selon les positionnements 7, les contextes, les grilles de lecture à l'œuvre, ces scènes sont vécues et perçues de façon différente. Il reste à comprendre pourquoi cette scène précisément a fait choc, à dépasser son apparente évidence. Par ailleurs, la deuxième question renvoie au lien ainsi fait, à la supposée continuité, entre un choc, un ressenti et un engagement. L'expression d'un sentiment d'injustice déborde le cadre militant, le choc émotionnel peut conduire à l'abattement et la résignation, des processus d'habituation et de routinisation se mettent en place même dans des contextes extrêmes ${ }^{8}$.

Le passage à l'engagement ne peut ainsi se comprendre sans inclure le rôle des passeurs, des réseaux de socialisation militante et la perception des répertoires d'action à disposition, ce qui a largement été étudié par la sociologie de l'engagement à partir de la reconstitution des trajectoires militantes dans divers contextes 9. C'est dans ce cadre que nous inscrirons la notion d'engagement ici utilisée pour désigner une inscription de longue durée dans des formes d'action politique collective ${ }^{10}$. Mentionnons aussi le rôle des grilles de

6. Traïni Ch., «Choc moral », in Fillieule O., Mathieu L. et C. Péchu (dir.), Dictionnaire des monvements sociaux, Presses de Sciences Po, 2009, pp. 101-107.

7. Caroline Guibet Lafaye montre la variabilité des liens entre expériences malheureuses et sentiment d'injustice dans le cadre d'une analyse des sentiments d'injustice en France. Guibet Lafaye C., Le Juste et l'Inacceptable. Les sentiments d'injustice contemporains et leurs raisons, Paris, Presses de l'Université Paris-Sorbonne, 2012, pp. 58-68.

8. Jutta Bakonyi note ces effets de routinisation de la violence en Somalie, et Laurent Gayer et Christophe Jaffrelot en Asie du Sud face à une « violence en miroir de l'État et des groupes armés privés ». Bakonyi J., "Government and the Quotidien: Everyday Experiences with Authority and Security in Somalia », communication pour le colloque Violence and Control in Civil Wars-Violent State Making, 7-9 décembre 2016, Hamburger Institut für Sozialforschung. Gayer L. et Jaffrelot Chr. "Conclusion », in Gayer L.et al., Milices armées d'Asie du Sud, Paris, Presses de Sciences Po, 2008, pp. 295-301. Le même processus de routinisation, qui n'exclut pas la peur au quotidien, a été abondamment étudié en Amérique latine : Green L., Fear as a Way of Life. Mayan Widows in Rural Guatemala, New York, Columbia University Press, 1999 ; Kees Koonings et Dirk Krujit (dir.), Societies of Fear. The Legacy of Civil War, Violence and Terror in Latin America, Londres, Zed Books, 1999.

9. Fréderic Sawicki et Johanna Siméant en font un des résultats transversaux en sociologie de l'engagement : Sawicki F. et J. Siméant, « Décloisonner la sociologie de l'engagement militant. Note critique sur quelques tendances récentes des travaux français ", Sociologie du travail, $\mathrm{n}^{\circ}$ 51, 2009, pp. 97-125. Molly Andrews, qui étudie quinze parcours militants de longue durée en Angleterre, parle, elle, d' " initiateurs ». Andrews M., Lifetimes of Commitment: Aging, Politics, Psychology, Cambridge, Cambridge University Press, 2008. Au Maghreb-Machrek et en Iran, Mounia Bennani-Chraibi et Olivier Fillieule notent les mêmes aspects : BennaniChraibi M. et O. Fillieule (dir.), Résistances et protestations dans les sociétés musulmanes, Paris, Presses de Sciences-Po, 2003. Pour les répertoires d'action collective : Tilly Ch., From Mobilization to Revolution, New York, Random House, 1978.

10. Un usage en sociologie politique de la notion d'engagement - voir notamment la définition 
lecture construites au sein des organisations politiques et la façon dont elles peuvent plus ou moins opérer des mises en sens des situations pour les militants. Cette fabrique du sens inclut des éléments émotionnels : émotions et sentiments de diverses sortes, vécus, inscrits dans les histoires des militants, encouragés et formatés par les organisations dont les cultures politiques comprennent des éléments affectifs ${ }^{11}$.

Nous proposons, donc, de reprendre à nouveaux frais ces questionnements sur le rapport entre sentiment d'injustice et engagement dans une double perspective. En premier lieu, en repartant de ce que nous savons : les observations in situ sur l'existence d'un choc moral conduisant à l'engagement sont rares et ce type d'approche encourt toujours le risque de la tautologie 12 (pourquoi y a-t-il eu choc moral ? parce que la situation a fait choc/était choquante). C'est donc bien plutôt l'énonciation de ce sentiment d'injustice dans le cadre d'un engagement, et notamment la manière dont certaines expériences sont mises à la source de ce sentiment, qui sont interrogées. L'analyse se concentre sur ces énonciations en contexte coercitif, avec la référence à des scènes d'oppression et de répression, en revenant sur la façon dont la violence politique et l'intensification émotionnelle qui y est liée influent sur ces expressions. Certaines des réflexions d'Axel Honneth sur l'expérience d'injustice et du mépris sont ainsi discutées, à partir de cette perspective décentrée. On s'appuie pour cela sur des recherches menées au Proche-Orient, sur nos propres recherches auprès de militants des différents courants politiques dans les Territoires palestiniens et en Jordanie ${ }^{13}$, ainsi que sur la littérature à disposition, en particulier sur la Syrie, sans exclure des comparaisons et des mises en perspective à partir d'autres contextes de violence politique ${ }^{14}$.

Une typologie des différents types de scènes et d'expériences d'injustice mises en avant par les militants en contexte coercitif est tout d'abord présentée. Elle met au jour la mobilisation de trois types d'expériences dans ces énonciations militantes : scène liminaire choquante, accumulation sur la durée d'expériences d'humiliation, de vexations et de discriminations marquant une situation structurelle, et récits transmis de l'injustice. Les modalités de ces

de Sawicki F. et J. Siméant (op. cit.) - qui se distingue d'autres usages en sociologie, par exemple comme mode d'inscription dans une action au sens général (Thévenot L., L'action au pluriel. Sociologie des régimes d'engagement, Paris, La Découverte, 2006), dans la lignée de la phénoménologie, ou, autre courant, comme processus de subjectivation dans l'action (Dubet Fr., Sociologie de l'expérience, Paris, Seuil, 1994).

11. Cefaï D. (dir.), Cultures politiques, Paris, Presses Universitaires de France, 2001, p. 17 et pp. 103-104.

12. Traïni Chr., op. cit.

13. Pour la présentation détaillée de ces enquêtes, voir Larzillière P., Être jeune en Palestine, Paris, Balland, 2004, et La Jordanie contestataire. Militants islamistes, nationalistes et communistes, Paris, Sindbad/Actes Sud, 2013. Des enquêtes complémentaires ont été menées dans les Territoires palestiniens en 2009.

14. Précisons ici que le contexte libanais, qui se situe pour partie dans une configuration politique différente par rapport à notre propos, ne sera pas au cœur de l'analyse. 
énonciations, les mises en sens et les légitimations à l'œuvre en rapport avec un engagement sont ensuite étudiées.

\section{Sentiment d'injustice en contexte coercitif : énonciations militantes}

" J'étais à une manifestation, à côté de moi, il y avait ce jeune sur les épaules d'un autre qui lançait les slogans, on s'approchait du check-point, et les soldats tiraient avec des balles en métal recouvertes de plastique, à un moment il a reçu une balle, dans la bouche 15 ». Ce militant palestinien revient sur la scène et souligne ensuite qu'elle a induit un tournant dans sa perception de la situation. Il s’est représenté précisément le soldat israélien, prenant son temps pour viser et attendre le moment où le jeune ouvrirait la bouche pour lancer le prochain slogan, et a basculé de ce fait du sentiment d'une répression aveugle et impersonnelle à la perception d'une malignité de l'ennemi, qui accentue pour lui l'arbitraire et l'injustice de la situation. Il associe ce basculement à une modification de son rapport à l'action politique ; de jeune suivant ses amis à la sortie des classes pour participer occasionnellement à des manifestations, il devient militant des jeunesses Fatah. Il en fait le point de départ d'un engagement à long terme sous une forme militante qu'il distingue des actions ponctuelles antécédentes qui, pour lui, ne revêtaient pas le même sens politique (même si on peut déjà y relever des formes de sensibilisation politique et de socialisation pré-militante, qui influent sur le positionnement et le vécu de l'acteur, mais peuvent aussi rester très temporaires et sans lien direct avec les organisations militantes).

La scène n'est pas la plus violente vécue par ce militant, mais elle correspond bien à la description d'un choc moral au sens où elle marque une prise de distance et une redéfinition de la situation : le geste répressif devient ici, pour lui, personnalisé, il prend de ce fait un autre sens et acquiert une intensité supplémentaire. De manière plus générale, la référence à un choc s'accorde avec le discours des militants qui mettent fréquemment en avant un événement initial, une scène choquante qu'ils décrivent avec émotion dans les entretiens. Dans le contexte palestinien, ces scènes renvoient habituellement à des interactions violentes et humiliantes avec l'armée israélienne, surtout lors de passages de check-points, scènes les concernant personnellement, ou tout autant, et peut-être même plus souvent parce qu'elles permettent au militant d'éviter de revenir sur des humiliations personnelles, scènes vues ${ }^{16}$. On peut notamment comparer sur ce point avec les énonciations militantes recueillies par Omar Carlier, qui évoque, dans le contexte de l'expérience coloniale en

15. Enquête de terrain en Cisjordanie, Ramallah, 2009.

16. Larzillière P., Etre jeune en Palestine, op. cit. " Le passage au barrage militaire », pp. 59-66. Voir également l'analyse du récit d'une de ces scènes fondatrices par Romani V., "Générations politiques et générations universitaires dans les Territoires palestiniens : nationalismes et dé-marxisation ", Revue internationale de politique comparée, vol. 16, n 2, 2009, p. 289. 
Algérie, la référence faite par les militants à ces scènes fondatrices, au « choc personnel ». En Tunisie, Michaël Bechir-Ayar montre le rapport entre engagement et traumatismes véhiculant un fort sentiment d'injustice sur lequel s'articule l'explication idéologique ${ }^{17}$.

Dans un tout autre contexte, celui de militants sikhs au Khalistan en Inde, Cynthia Keppley Mahmood analyse également la façon dont des scènes liminaires vues sont présentées par les militants comme déterminantes dans leur passage à un engagement violent ${ }^{18}$.

Ces scènes cristallisent l'oppression, parce que la dignité des personnes respectées est remise en cause, parce qu'elles ont pour victimes des personnes considérées comme particulièrement vulnérables (et inoffensives), ou représentent une humiliation personnelle. Il ne s'agit pas d'un changement brusque de situation, la situation d'oppression est la même avant et après, mais il y a effectivement changement de perception par la disparition de certains repères - avec l'humiliation des personnes de référence - , ou par le passage à un degré supplémentaire de répression dans une situation d'oppression, à travers ces victimes particulières ${ }^{19}$.

Ce point invite d'ailleurs à reconsidérer une des caractéristiques des contextes coercitifs : l'incertitude générée par l'imprévisibilité de la réaction de la force occupante ou de l'État, laquelle fait partie intégrante de la coercition ${ }^{20}$. Le retour sur ces scènes qui marquent les militants montre que, s'il y a incertitude, et mise à merci, il y a aussi un régime répressif « usuel », puisque des «sorties » de cadre peuvent être repérées ${ }^{21}$.

Toutefois, ces sorties de cadre ne sont pas les seules à être mentionnées, et une scène fondatrice n'est pas toujours mobilisée pour rendre compte de l'émergence d'un sentiment d'injustice, une des difficultés de la notion étant l'idée d'un changement brusque de situation ou de perception. D'autres types

17. Carlier O., Entre nation et Jihad: Histoire sociale des radicalismes algériens, Paris, Presses de la FNSP, 1995, p. 56. Michaël Bechir-Ayari, S'engager en contexte autoritaire. Gauchistes et islamistes dans la Tunisie indépendante, thèse de doctorat, Université d'Aix-en-Provence, 2009.

18. Keppley Mahmood C., Fighting for Faith and Nation. Dialogues with Sikh Militants, University of Pennsylvania Press, 1996.

19. Dans un autre type de contexte, Kotronaki Loukia et Seferiades Seraphim montrent comment un «événement non normalisé de violence répressive » peut induire ce choc et entendent parlà « un type d'événement répressif qui n’est pas assimilé et inscrit dans l'imaginaire collectif en tant qu'expression conventionnelle et/ou convenue du "répertoire répressif”, et qui met en question autant les schémas d'injustice tolérés que les “routines contestataires” ». Loukia K. et S. Seraphim, "Sur les sentiers de la colère : l'espace-temps d'une révolte (Athènes, décembre 2008) », Actuel Marx, vol. 48, no 2, 2010, pp. 152-165.

20. Les passages aux check-points en particulier marquent l'entrée dans ces zones d'incertitude qui signent la domination. Larzillière P., Etre jeune en Palestine, op. cit. ; Pommerolle M.-E. et F. Vairel, «S’engager en situation de contrainte », Genèses, vol. 77, n 4, 2009, p. 3.

21. Bakonyi J., Government and the Quotidien, op. cit. 
d'expérience sont mis en avant. Non pas un choc, mais, tout au contraire, une usure, une « situation » dont il est donné de multiples exemples, une multitude de petites expériences dont l'accumulation produit un sentiment d'injustice comme si elle donnait corps, donnait à ressentir une injustice structurelle. Dans le contexte syrien, des volontaires d'associations de bienfaisance, impliqués dans le soulèvement de 2011, mettent en avant un sentiment d'injustice nourri de multiples épisodes de discrimination entre sunnites et alaouites ${ }^{22}$. Omar Carlier souligne aussi cet aspect en Algérie : choc ou « accumulation de petites vexations de la vie quotidienne 23 ». En Jordanie, les militants de différentes tendances, islamistes, nationalistes ou communistes, mettent aussi à la source de leur engagement un sentiment d'injustice, mais ils l'associent moins à des scènes fondatrices qu'à des "situations » injustes : situation historique des Palestiniens (qu'ils le soient ou non) et pauvreté, plus spécifiquement une pauvreté rurale ${ }^{24}$. Dans la présentation de ce sentiment d'injustice lié à des situations générales, des liens sont faits cependant avec des expériences personnelles : des militants qui, plus jeunes, habitaient près de la frontière et ont vu de près l'exil palestinien et les affrontements, ou la dureté du travail des champs, loin des représentations pastorales avec la mention, par exemple, d'un grand-père courbé sous sa maigre récolte qu'il porte au marché sur son dos. La confrontation à une violence politique directe ne représente, donc, ici qu'une part des expériences référées à un sentiment d'injustice. Dans le cas jordanien, le sentiment d'injustice semble plus indéterminé ou, en tout cas, est considéré comme tel par les militants, même si dans leur présentation apparaît une idée implicite de ce que serait une société juste. La référence à la pauvreté est, d'ailleurs, une donnée classique de la construction d'un sentiment d'injustice. Elle renvoie aussi à une sorte de cadrage rétrospectif, nous y reviendrons : ici, la mise en forme par les organisations post-engagement est plus centrale, et les expériences de répression directe constituant des moments charnières sont en général postérieures à l'engagement.

Le registre coercitif influe : en Jordanie, les militants se trouvent face à un régime autoritaire libéral, et non une situation d'occupation, et la répression y est moins forte et moins visible. L'autoritarisme libéral, une forme hybride de régime, n'exclut pas l'existence d'institutions démocratiques, mais celles-ci ne servent ni à l'alternance du pouvoir ni à définir les politiques publiques ${ }^{25}$. Les

22. Ruiz de Elvira L.et S. Belhadj, "Sectarianism and Civil Conflict in Syria: Reconfigurations of a Persistent Issue”, in Rivetti P.et H. Kraetzschmar (dir.), Islamists and the Politics of the Arab Uprisings. Governance, Pluralisation and Contention. Transformation of Political Islam, Edinburgh University Press, 2018.

23. Carlier O., Entre nation et jihad, op. cit., p. 56.

24. Larzillière P., La Jordanie contestataire, op. cit.

25. Juan Linz avait inclus dans ses catégorisations des régimes autoritaires les régimes «à pluralisme limité » (Linz J. J., Totalitarian and Authoritarian Regimes, Boulder Londres, Lynne Rienner Publishers, 2000 [1975], voir la nouvelle introduction et p. 159 et suiv.). Depuis, une vaste littérature s'est intéressée aux formes des régimes hybrides et à l'autoritarisme libéral, voir notamment Diamond L., “Thinking About Hybrid Regimes”, Journal of Democracy, vol. 13, $\mathrm{n}^{\circ}$ 2, 2002, pp. 21-35; O’Donnell G.et Ph. C. Schmitter, Transitions from Authoritarian Rule: Tentative Conclusions, vol. 4, Baltimore, Johns Hopkins University 
gouvernants y demeurent indépendants des gouvernés ${ }^{26}$, ainsi dans le cas jordanien, la monarchie, bien qu'officiellement constitutionnelle, se situe en dehors du système représentatif tout en disposant des quasi pleins pouvoirs. Cela ne signifie pas pour autant que ces institutions, élections et parlement, n'ont aucun rôle. En Jordanie, les élections - souvent analysées comme des formes d'élections autoritaires - plutôt que d'être une base pour l'opposition, permettent d'établir des élites intégrées et de mettre en place des structures de patronage et de clientélisme. Aussi, est-ce dans d'autres arènes que se joue l'opposition au régime. Par ailleurs, l'importance de l'appareil sécuritaire a des effets dissuasifs en amont, contribuant à l'évitement du politique, avec censure et autocensure dans les médias.

En dernier lieu, un troisième type d'expérience est mentionné : les récits transmis de l'injustice. Ainsi, si l'on reprend l'expérience palestinienne au Proche-Orient : les militants indiquent, tout particulièrement en diaspora, combien ils ont été marqués par les récits de l'exil de 1948 et 1967, tels qu'ils sont transmis par les grands-parents ou les parents qui narrent la violence de l'exil, mais ne cessent aussi de souligner le décalage entre leur situation actuelle, leurs conditions de vie dans les camps de réfugiés, et leur situation antérieure dans la Palestine de 1948. Ces récits sont souvent mobilisés par les militants lorsqu'ils veulent rendre compte de leur passage à l'engagement et de l'injustice du sort fait aux Palestiniens 27. Ainsi, en ce qui concerne les cadres de l'injustice, si l'on relève toujours les cadrages des organisations et le façonnage ou pré-formatage institutionnel, on oublie combien les sources des mises en sens sont diverses, et la façon dont les récits transmis d'expériences parentales ou grand-parentales influent 28.

Press, 1986 ; Hermet G., « Un régime à pluralisme limité ? À propos de la gouvernance démocratique ", Revue française de science politique, vol. 54, n 1 , 2004, pp. 159-178. Une évolution notable dans cette littérature depuis les années 2000 est l'éloignement des visions en termes de transition démocratique. Ce que montrent les analyses de ces régimes, et à nouveau le cas du régime jordanien, c'est la compatibilité entre l'instauration de certaines institutions démocratiques et l'autoritarisme. Contrairement aux attentes des théories classiques de la transition démocratique, l'ouverture démocratique peut simplement signifier l'intégration de ces institutions dans le système autoritaire. Et les évolutions politiques dépendent bien plutôt d'un jeu complexe entre les différentes arènes politiques dont ces institutions démocratiques ne sont qu'une part. Cf. Albrecht H. (dir.), Contentious Politics in the Middle East: Political Opposition under Authoritarianism, Gainesville, University of Florida, 2009, p. 2 ; Camau M., « Remarques sur la consolidation autoritaire et ses limites » in Boutaleb A., Ferrié J.-N. et B. Rey (dir.), L'Autoritarisme dans le monde arabe, Le Caire, CEDEJ, 2005, pp. 9-51; Dabène O., Geisser V. et G. Massardier (dir.), Autoritarismes démocratiques et démocraties autoritaires an XXIe siècle : Convergences Nord-Sud, Paris, La Découverte, 2008, p. 21 ; Larzillière P., "Political Commitment under an Authoritarian Regime: Professional Associations and Islamist Movement as Alternative Arenas in Jordan”, International Journal of Conflict and Violence, vol. 6, ${ }^{\circ} 1,2012$, pp. 11-25.

26. Ferrié J.-N., « Dispositifs autoritaires et changements politiques. Les cas de l'Égypte et du Maroc », Revue internationale de politique comparée, vol. 19, n 4, 2012, pp. 93-110.

27. Napolitano V., S'engager à Yarmouk. Sociologie de la militance palestinienne en Syrie, thèse de doctorat, EHESS, 2015. Al Husseini J. et A. Signoles (dir.), Les Palestiniens entre État et diaspora: le temps des incertitudes, Paris, Karthala/IISMM-EHESS, 2012. Larzillière P., La Jordanie contestataire, op. cit., p. 50. 
Trois types d'expérience sont, donc, ici mobilisés pour rendre compte d'un sentiment d'injustice à l'origine d'un engagement, scène liminaire et choc n'étant que l'une d'entre elles. Ces expériences mettent en exergue la prégnance d'un contexte. Le récit personnalise l'expérience, mais pas toujours du fait de l'importance de la référence à un destin collectif pour « surmonter » ces expériences, et au moins les mettre en sens. Cet aspect apparaît, en particulier, dans les récits des humiliations et des violences vécues lors des passages de check-points de l'armée israélienne : le récit de l'expérience individuelle vécue est immédiatement réinscrit dans une référence à un destin collectif, qui introduit une distanciation par rapport à l'humiliation personnelle. Dans tous les cas, reste à la source la violence de l'interpellation et des émotions générées.

Si l'on met ces expériences en contextes coercitifs ou autoritaires lorsque la répression violente est au cœur du régime politique - en regard avec l'analyse des sentiments d'injustice en contextes démocratiques occidentaux actuels, que peut-on noter ? Dans ces contextes, deux éléments sont mis en avant pour l'apparition de ces sentiments d'injustice : l'importance des dispositifs de sensibilisation des organisations qui génèrent des émotions autour des causes tout d'abord ${ }^{29}$, et le caractère diffus et imprécis du sentiment d'injustice ${ }^{30}$.

En contexte coercitif, on pourrait raisonner à l'inverse : les émotions sont là, et la domination n'apparaît pas de manière diffuse ou imprécise, mais déjà en partie cristallisée : l'occupant, le régime, les institutions oppressives, etc. Le travail de sensibilisation des organisations politiques est donc ici moins essentiel (et c'est bien plutôt de pouvoir « échapper » d'une manière ou d'une autre à ces situations, ne pas être "pris " par, qui semble faire l'objet d'un travail). L'exemple jordanien est à cet égard instructif comme se situant à l'intermédiaire des deux modèles. Les liens entre type de violence politique et de régime, et modes d'expression d'un sentiment d'injustice ressortent ici. La répression concerne-t-elle spécifiquement les militants, est-elle plus large ? 31

28. Voir aussi sur la question des transmissions traumatiques des militants : Bechir-Ayari M., S'engager en contexte autoritaire, op. cit.

29. Christophe Traïni et Johanna Siméant définissent les dispositifs de sensibilisation comme «l'ensemble des supports matériels, des agencements d'objets, des mises en scène que les militants déploient afin de susciter des réactions affectives qui prédisposent ceux qui les éprouvent à s'engager ou à soutenir la cause défendue ». Cela peut comprendre par exemple la diffusion d'un répertoire artistique autour de la cause : chants, organisations de concerts, performances théâtrales de rue, etc., la production de vidéos et de documentation visant à provoquer le choc et l'indignation, des commémorations mémorielles, etc. Il est à noter que ces dispositifs ne visent pas seulement de nouveaux sympathisants, mais aussi l'affectivité des militants eux-mêmes. Traïni C. et J. Siméant, « Pourquoi et comment sensibiliser à la cause ? », in Traïni C. (dir.), Émotions... Mobilisations!, Paris, Presses de Sciences Po, 2009, p. 13.

30. Dubet Fr., Ce qui nous unit. Discriminations, égalité et reconnaissance, Paris, Seuil, 2016. Guibet-Lafaye C., Le Juste et l'Inacceptable, op. cit.

31. Combes H. et $\mathrm{O}$. Fillieule, «De la répression considérée dans ses rapports à l'activité protestataire. Modèles structuraux et interactions stratégiques ", Revue française de science politique, vol. 61, n 6, 2011, pp. 1047-1072. 
S'agit-il de mesures répressives ponctuelles ou, au contraire, d'une donnée structurelle du régime ? Se donne-t-elle à voir en des formes explicites, ou se traduit-elle par des appareils de sécurité travaillant dans l'ombre et en amont, à décourager la dissidence ? Tous ces éléments pèsent. Pour autant, souligner ce point ne revient pas à faire du sentiment d'injustice une sorte d'émotion liée sui generis à certaines situations. De fait, les énonciations militantes d'un sentiment d'injustice se situent au croisement de plusieurs registres - émotionnel, moral et de légitimation - et si le travail de sensibilisation des organisations est moindre, il retrouve toute son importance dans la définition des cadrages et surtout des possibilités de lutte.

\section{Sentiment d'injustice et cadrages normatifs}

Par rapport au panel d'émotions que peut susciter la confrontation à la violence politique, traumas de différentes formes, colère, volonté de vengeance ou sentiment d'humiliation, le sentiment d'injustice présente une spécificité. En premier lieu, le vocabulaire utilisé est en lui-même important, on parle de sentiment d'injustice et on peut s'appuyer ici sur les distinctions effectuées par Isabelle Sommier autour des affects liés aux mobilisations, en restant dans une perspective résolument sociologique. Un des enjeux, en effet, dès qu'on aborde la question des émotions politiques, est d'éviter l'écueil de la psychologie des foules et les «disqualifications morales » liées à ces représentations de contestataires irrationnels ${ }^{32}$. En reprenant les définitions d'André Akoun et Pierre Ansart, Isabelle Sommier différencie les sentiments des affects, des émotions et des passions et caractérise le sentiment par sa longue durée (et son inscription dans une socialisation, ce qui vaut cependant aussi pour les émotions) 33 .

Le sentiment d'injustice possède une seconde spécificité, qui renvoie au contenu moral associé au caractère émotionnel : il s'inscrit dans la référence à des normes sociales et tend vers un sens commun et la représentation d'un bien commun ${ }^{34}$. Le sentiment d'injustice constitue de ce fait une mise en sens qui peut, en partie d'ailleurs, être infra argumentative et représenter un hori-

32. Voir sur ce point : Goodwin J., Jasper J. et F. Polletta, Passionate Politics. Emotions and Social Movements, The University of Chicago Press, 2001 ; Sommier I., "Les états affectifs ou la dimension affectuelle des mouvements sociaux ", in Fillieule O., Agrikoliansky E. et I. Sommier (dir.), Penser les mouvements sociaux. Conflits sociaux et contestations dans les sociétés contemporaines, Paris, La Découverte, 2010, pp.185-202 ; C. Traïni, Choc moral, op. cit., p. 101. En ce sens, plutôt qu'au « retour » des passions politiques elles-mêmes, mis en avant par Pierre Hassner, on assisterait à un renouveau de la légitimité des explorations scientifiques de la question des émotions. Hassner P., La revanche des passions. Métamorphoses de la violence et crise du politique, Paris, Fayard, 2015.

33. Sommier I., « Les états affectifs... », op. cit., p. 192.

34. Lorsqu'il s'interroge sur la nature du sentiment d'injustice de Michael Kohlhaas dans la fameuse nouvelle de Kleist, François Chazel relève à nouveau cette dimension proprement morale. Chazel Fr., "Sentiment d'injustice, lutte pour le droit et rationalité axiologique : variations sur le "cas" de Michael Kohlhaas", Droit et société, vol. 55, n³, 2003, pp. 769-780. 
zon plutôt qu'une énonciation précise. Mais l'expression même d'un sentiment d'injustice se réfère a priori à une intersubjectivité morale et, de ce fait, à l'idée qu'il puisse être partagé, et même qu'il dut l'être. Il ne s'agit pas seulement ici d'empathie ou de la diffusion d'émotions lors d'expériences collectives ${ }^{35}$. Car la référence en creux à des normes par la dénonciation de leur transgression est un appel à une indignation partagée, et il est donc peu étonnant que ce soit précisément ce sentiment que mobilisent les militants dans la légitimation de leur engagement. Il inclut dans son expression une demande de réparation et représente une justification en soi. Lorsqu'une militante des jeunesses Fatah évoque une femme enceinte brutalisée à un check-point, et fait de cette scène qui la choque le point de départ de sa volonté d'engagement ${ }^{36}$, elle pointe en creux plusieurs transgressions. Au-delà de l'oppression « routinière » des passages plus ou moins difficiles aux check-points, une sortie de cadre a eu lieu dont le caractère genré renforce l'impact : violence politique contre une femme, et plus encore violence politique contre une mère. Les représentations de genre associent les femmes à des figures de paix ou de victimes dans les conflits, figures non menaçantes, et rendent les violences qui leur sont faites dans ce cadre plus transgressives. Ici, cet aspect est davantage renforcé par la figure de femme enceinte, figure de femme particulièrement à merci où une double vie est en jeu, et figure de mère. Les violences faites aux mères sont souvent mises en avant comme des expériences particulièrement choquantes, ce que l'on peut lier aux constructions de genre autour du rôle maternel, à deux niveaux. Dans le cadre de luttes nationales, des constructions symboliques spécifiques sont effectuées, avec des associations figuratives entre la terre et la mère, entre le peuple et la mère ${ }^{37}$; ainsi, violenter une mère, c'est exercer une violence individuelle, mais aussi, symboliquement, profaner une figure du collectif. Plus largement, souligner les violences faites aux mères, c'est aussi s'appuyer sur les constructions de genre dans les perceptions occidentales de ces conflits, où ce type de violences apparaît comme particulièrement arbitraire, illégitime et choquant, et faire ainsi appel à une indignation dépassant les enjeux du conflit national et renforçant la possibilité d'empathie. Joanna Allan le montre dans le cas du conflit du Sahara occidental, en restituant le témoignage d'une militante nationaliste kidnappée et torturée par la police marocaine. La militante souligne qu'elle est aussi une mère et qu'en ce sens son destin illustre le caractère déshumanisé de l'agresseur prêt à toutes les transgressions 38 .

35. Nous nous concentrons ici sur l'expression d'un sentiment d'injustice, mais d'autres registres émotionnels (joie, haine, enchantement, désenchantement ou amertume, entre autres) peuvent aussi être mentionnés par les militants en référence à leur engagement. Moins pour le légitimer cette fois que pour évoquer leurs ressentis vis-à-vis de leurs parcours ou lors des actions collectives. "Les beaux jours » souligne ainsi un ancien militant communiste jordanien en référence à l'enchantement de l'action collective et d'un entre-soi fraternel, malgré la violence de la répression.

36. Enquête de terrain en Cisjordanie, Ramallah, 2009.

37. Larzillière P., Etre jeune en Palestine, op. cit.

38. Allan J., "Orientalisme, genre, répression et contestation au Sahara Occidental », in Boulay S.et Fr. Correale (dir.), Sahara Occidental : conflit oublié, population en mouvement, Tours, 
Le sentiment d'injustice ajoute, donc, à l'expression émotionnelle une inscription morale et en creux la référence à des normes, ici transgressées. Les analyses de l'indignation comme rejet de l'injustice montrent cette articulation entre une émotion, la colère, et une sensibilité morale face à ce qui est perçu comme injuste et indigne. Ces normes du juste peuvent recouvrir des contenus variables, et renvoyer parfois à l'identification à des groupes particuliers, dans un "eux et nous », aussi ces analyses insistent-elles sur la nécessaire explicitation de ces normes dans une démarche délibérative, au-delà d'un ressenti ${ }^{39}$. Axel Honneth analyse cette expérience de l'injustice, sous l'angle du mépris [Missachtung] et du déni de reconnaissance. Il propose une typologie qui distingue spécifiquement l'expérience d'injustice associée à la violence, et permet de rendre compte de son impact particulier. Trois formes de remise en cause de la relation intersubjective au cœur de la socialisation sont ainsi déclinées : les sévices corporels, les blessures morales («modes de mépris personnel dont un sujet est victime lorsqu'il se trouve structurellement exclu de certains droits au sein de la société $40 »)$, et le dénigrement social et culturel portant sur certains modes de vie individuels ou collectifs, leur déniant toute valeur sociale et représentant une atteinte à la dignité. La première forme, les atteintes à l'intégrité physique, nous intéresse plus particulièrement ici. Axel Honneth montre qu'elles revêtent un caractère fondamental en ce qu'elles "nient la capacité même du sujet à disposer librement de son propre corps ». Contrairement aux autres formes, appuyées sur des droits dont la définition est historiquement et culturellement située, cette expérience du mépris lié aux sévices physiques transcende en partie ces cadres de référence. Car, si le rapport au corps ainsi mis à mal se construit lui aussi dans une intersubjectivité, il repose sur « des présupposés affectifs qui obéissent à une logique pour une grande part invariante, de l'équilibre intersubjectif entre fusion et démarcation psychique ${ }^{41} »$. La torture, par exemple, représente un tel déni de ce rapport fondamental, que le sujet, privé de toute autonomie et pris dans une souffrance qui détruit toute relation de communication ${ }^{42}$, peut en perdre le sentiment de sa propre réalité. Certes, il existe aussi des normes sociales autour de la violence et le corps violenté et souffrant reste inscrit dans des formes sociales situées, voire revêt un sens politique ${ }^{43}$. Mais il reste que ce type d'injustice renvoie à des expériences structurelles dont le caractère choquant

Presses universitaires François Rabelais, 2018, pp. 209-222. Pour une analyse des enjeux de la mise en visibilité des corps féminins souffrants dans les productions militantes sahraouies, voir Boulay S., "Corps, lutte pacifique et création sur le Web », in S. Boulay et F. Correale, op. cit., pp. 222-241.

39. Foessel M., "Les raisons de la colère ", Esprit, n 3, mars-avril 2016, pp. 43-53. Cordell C., "L'indignation entre pitié et dégoût : les ambiguités d'une émotion morale », Raisons politiques, vol. $65, \mathrm{n}^{\circ} 1,2017$, pp. 67-90.

40. Honneth A., Kampf um Anerkennung, Frankfurt am Main, Suhrkamp Verlag, 1992. Éd. fra. : La Lutte pour la reconnaissance, Paris, éditions du Cerf, 2000, p. 163. Trad. de Pierre Rusch.

41. Ibid.

42. Sur ce processus de désubjectivation sous la torture, voir notamment Terestchenko M., « De l'utilité de la torture ? Les sociétés démocratiques peuvent-elles rester des sociétés décentes ? ", Revue du MAUSS, vol. 28, n 2, 2006, pp. 337-366. 
paraît particulièrement universalisable et, donc, de même l'indignation ou l'appel à l'indignation qu'elles suscitent.

Pour autant, un deuxième aspect de la réflexion d'Axel Honneth n'est pas ici corroboré par la façon dont les militants se réfèrent au sentiment d'injustice. Il a, d'ailleurs, déjà été remis en cause par Caroline Guibet-Lafaye dans son analyse des expressions du sentiment d'injustice en France, soulignant qu'Axel Honneth oppose sentiment et cohérence argumentative, et suppose que les dominés expriment un sentiment d'injustice non articulé à des représentations morales abstraites. Caroline Guibet-Lafaye montre, au contraire, que « les individus, y compris placés dans des positions de désavantage social, expriment des représentations morales, articulées de façon cohérente, voire systématique (...). Pressés par l'exigence de donner sens à leur existence et à leur position dans l'ordre social, ils se voient contraints d'essayer de comprendre ou d'interpréter le fonctionnement de cet ordre 44 ». Or, dans son article de 1981 (pour l'édition allemande), Axel Honneth renvoie la notion de sentiment d'injustice du côté de « la morale sociale de classes défavorisées » qui, du fait de la «pression directe que le problème exerce sur celles-ci » et de leur trop grande implication émotionnelle dans les situations à évaluer, " [n’]offre [qu'] un ensemble disparate de revendications réactives, tandis que les conceptions élaborées de la justice évaluent les faits sociaux dans un système de référence cohérent 45 ». Notons que dans La lutte pour la reconnaissance (1992 pour l'édition allemande), la position d'Axel Honneth se nuance. Les sentiments d'injustice y deviennent une "matière première émotionnelle ", qui n'est plus référée spécifiquement à des classes défavorisées trop impliquées. La distinction reste nette entre ces expressions émotionnelles et la référence à un système moral cohérent, mais il considère que, dans certains contextes politiques et culturels, ces sentiments peuvent acquérir un "potentiel cognitif » et constituer une première indication de transgression d'un consensus moral tacite, à condition d'être réinscrits dans une sémantique morale collective ${ }^{46}$.

En contexte coercitif, si, comme il a été montré précédemment, le sentiment d'injustice est référé à trois types d'expériences (scène liminaire choquante, usure d'une situation aux humiliations répétitives et récits transmis de l'injustice), et si ces expériences sont souvent mises en avant avec émotion, on

43. Memmi D., «Introduction : la dimension corporelle de l'activité sociale ", Sociétés contemporaines, $\mathrm{n}^{\circ}$ 31, 1998, pp. 5-14. Mauss M., "Les techniques du corps ", Journal de Psychologie, vol. XXXII, n 3-4, mars-avril 1936, pp. 271-293.

44. Guibet-Lafaye C., "Domination sociale et représentations du juste », Revne européenne des sciences sociales, vol. $50, \mathrm{n}^{\circ} 2,2012, \mathrm{p} .166$.

45. Honneth A., «Moralbewusstsein und soziale Klassenherrschaft. Einige Schwierigkeiten in der Analyse normativer Handlungspotentiale ", Leviathan. Zeitschrift für soziale Wissenschaft, vol. 9, $\mathrm{n}^{\circ}$ 3-4, 1981, pp. 556-570. Éd. fra. : «Conscience morale et domination de classe. De quelques difficultés dans l'analyse des potentiels normatifs d'action ", in Honneth A., La Société du mépris, Paris, La Découverte/Poche, 2006. Trad. de Pierre Rusch, p. 208 et 209.

46. Honneth A., La Lutte pour la reconnaissance, op. cit., p. 169 et 170. 
ne peut considérer qu'il y ait opposition entre ce registre émotionnel et la cohérence argumentative. Dans l'énonciation de ce sentiment d'injustice, les militants circulent entre l'expression d'un sentiment appelant l'empathie et la référence à des principes axiologiques abstraits ou à la représentation d'une société juste ${ }^{47}$. L'expérience personnelle mobilisée et l'expression émotionnelle qui lui est liée sont mises en regard avec, par exemple, des considérations sur les inégalités sociales, la répartition des revenus et des terres, les discriminations selon les catégorisations sociales, nationales ou religieuses, le droit d'expression dans la société, ou des réflexions morales en particulier sur le respect de la dignité humaine. Des comparaisons sont faites entre ce qui est et ce qui devrait être selon ces principes, et des éléments de réflexivité apparaissent lorsque les militants se situent dans un système général et pas nécessairement comme les plus défavorisés à l'intérieur de celui-ci. La particularité du sentiment d'injustice réside précisément dans l'articulation de ces registres. Et la mise en récit rétrospective conduit plutôt à une mise en cohérence, dont les expressions émotionnelles font partie, dans une présentation qui prend en compte le contexte d'énonciation pour mettre en avant ce qui apparaît au militant comme le plus à même de convaincre l'interlocuteur. Une présentation qui s'inscrit d'emblée dans des codes partagés - ainsi les scènes liminaires présentent des récurrences qui n'ont pas seulement à voir avec le fait de vivre dans un même contexte coercitif. Les scènes les plus propres à soulever l'indignation sont sélectionnées et renvoient à des narrations et des références collectives qui ne sont pas uniquement celles des organisations politiques. Des mises en cohérence sont effectuées, inscrites dans des constructions collectives de l'injustice - la référence à une communauté de destin par exemple - et peuvent apparaître en dehors des cadrages politiques.

\section{Sentiment d'injustice et engagement}

Reste, enfin, la question de l'articulation entre cette expression d'un sentiment d'injustice et l'engagement. La référence à un sentiment d'injustice est un mode d'explication massivement utilisé pour rendre compte des mobilisations, et, parfois, cet usage du sentiment d'injustice comme variable explicative n'est qu'une autre manière de désigner une frustration relative. On peut prendre ici pour exemple la façon dont la montée des Frères musulmans au sein du monde arabe, dans les années 1980, avait largement été interprétée en termes de mouvements de diplômés-chômeurs : ces nouvelles générations de jeunes diplômés, souvent premiers diplômés dans leurs familles, qui, contrai-

47. Lorsqu'elle analyse la référence au sentiment d'injustice des récupérateurs de déchets d'Istanbul et leurs tentatives de mobilisation, Bénédicte Florin identifie ainsi un double registre : celui de l'indignité de la situation qui leur est faite et l'inscription dans un registre plus général autour d'une vision sociale écologique, qui leur permet de revaloriser et donner une nouvelle légitimation à leur activité. Florin B., «De l'indignité à l'indignation : petites luttes, résistances quotidiennes et tentatives de mobilisation des récupérateurs de déchets à Istanbul », Cultures EConflits, $\mathrm{n}^{\circ}$ 101, 2016, pp. 99-119. 
rement à leurs attentes, ne trouvaient pas de travail qualifié. Ce type d'explications est à nouveau mobilisé dans le premier temps des soulèvements arabes de 2011, où Mohammed Bouazizi, le jeune vendeur de fruits et légumes tunisien qui s'immole par le feu fin 2010, après une longue suite d'avanies policières et la confiscation de sa marchandise, est tout d'abord faussement considéré comme un de ces diplômés et une figure du déclassement ${ }^{48}$.

Or, les explications des mobilisations en termes de frustration ou de frustration relative ${ }^{49}$, lorsqu'elles sont considérées comme des facteurs suffisants et intrinsèques, tendent à stabiliser et simplifier les processus : on trouvera toujours assez de frustration ou de frustration relative pour rendre compte d'une mobilisation ou d'un engagement ${ }^{50}$. Ce type d'explications repose sur une «illusion étiologique » au sens où elle consiste à rendre compte de l'action collective en remontant en amont aux conditions préexistantes qui l'auraient permise ${ }^{51}$. Dès lors, l'identité du groupe ou de l'individu, ici «frustré », est considérée comme une donnée stable et réifiée, antérieure au passage à l'action ${ }^{52}$. Ni les évolutions au cours de l'action ni les nombreux cas où, dans les mêmes conditions, l'acteur est conduit à l'apathie plutôt qu'à l'action n'apparaissent.

La référence au sentiment d'injustice ne peut, ainsi, ni être simplement rabattue sur une analyse en termes de frustration, ni être utilisée seule comme une variable explicative de l'engagement. Par rapport à la théorisation d'Axel Honneth - et même s'il questionne la façon dont cette expérience «peut envahir la vie affective des sujets humains au point de les jeter dans la résis-

48. Bennani-Chraïbi M. et O. Fillieule, «Pour une sociologie des situations révolutionnaires. Retour sur les révoltes arabes ", Revue française de science politique, vol. 62, $\mathrm{n}^{\circ}$ 5, 2012, p. 773. Pierre Blavier, «Sociogenèse de la révolution tunisienne : expansion scolaire, chômage et inégalités régionales ", Actes de la recherche en sciences sociales, vol. 211-212, n 1, 2016, pp. 55-71.

49. La théorie de « la courbe en J » de James Davies, qui ramène le passage à l'action à l'existence d'un "écart intolérable » entre satisfaction escomptée des besoins et satisfaction réelle, en constitue un bon exemple (Davies J., When Men Revolt and Why, New York, The Free Press, 1970, pp. 134-147). Reprise et systématisée par Ted Gurr, cette analyse en termes de frustration relative tend à verser dans un psychologisme réifiant, comme le montre Michel Wieviorka, et à mettre de côté les conditions politiques de la rébellion (Gurr T., Why Men Rebel, Princeton, Princeton University Press, 1970 ; Wieviorka M., Sociétés et terrorisme, Paris, Fayard, 1988, p. 472). Bennani-Chraïbi M. et O. Fillieule reviennent sur cette illusion étiologique en sociologie des révolutions et, en particulier, dans l'analyse des situations révolutionnaires de 2011. Bennani-Chraibi M. et O. Fillieule, « Pour une sociologie des situations révolutionnaires... », op. cit., p. 769.

50. Voir sur ce point, la critique développée par les théories de la mobilisation des ressources, qui soulignent que ces frustrations ne sont qu'un élément secondaire des mobilisations, voire sont parfois constituées rétroactivement par rapport aux mouvements : McCarthy J. D.et Z. N. Mayer, "Mobilization and Social Movements: A Partial Theory", The American Journal of Sociology, vol. 82, n 6, mai 1977, p. 1215 ; Jasper J., "Social Movement Theory Today: Toward a Theory of Action?”, Sociology Compass, vol. 11, n 4, 2010, pp. 965-976.

51. Dobry M., Sociologie des crises politiques, Paris, Presses de Sciences-Po, 1992, pp. 48-52.

52. Bigo D. et R. Brousse, «Identité et action collective » in « L'action collective : terrains d'analyse ", Cultures $\&$ conflits, $\mathrm{n}^{\circ} 12$, hiver 1993, p. 6. 
tance et l'affrontement social, autrement dit dans une lutte pour la reconnaissance 53 » - il reste à ajouter que pour « jeter les acteurs sociaux dans la résistance », il faut bien qu'une action leur paraisse possible, ou même, comme le souligne Axel Honneth lui-même, «que l'expérience du mépris (...) puisse s'articuler sur un mouvement social déjà constitué 54 », même si les modalités de cette articulation ne constituent pas le cœur de sa réflexion qui n’inclut pas de théorie de l'action.

Les recherches menées dans différents contextes de violence politique, en particulier sur le passage à l'engagement armé en Amérique latine 55, soulignent la non-continuité entre émotion et action : selon les contextes, les mêmes émotions politiques ne conduisent pas à l'action, il faut pour cela des opportunités, la proximité avec des groupes et des idéologies orientant ces émotions vers l'action, comme condition nécessaire (mais non suffisante). Enzo Nussio fait un pas de plus dans cette direction en montrant, à partir de recherches menées en Colombie, que les chocs moraux eux-mêmes ne peuvent être considérés comme des expériences premières, émotions et idéologies y sont déjà intriquées. Et lorsque Jocelyn Viterna retrace les trajectoires de femmes guérilleras au Salvador, elle analyse la façon dont ces femmes lient leur socialisation au parti et la lutte armée avec la possibilité de parler de l'injustice, de partager une compréhension de l'injustice et l'intégration d'un système de valeurs ${ }^{56}$.

En effet, comme on l'a vu précédemment, on ne peut distinguer complètement un avant et un après : une expérience de l'injustice vécue individuellement qui conduirait ensuite à un engagement, même si les militants font le plus souvent cette distinction dans une mise en récit rétrospective. La description même de cette expérience comme expérience d'injustice renvoie à des cadres et des références collectifs, et il ne peut guère en être autrement pour un sentiment moral nécessairement inscrit, de manière plus ou moins explicite, dans un horizon normatif. Cela n'enlève rien au caractère fondamental de l'expérience d'injustice liée à une violence, ici politique, remettant en cause la possibilité même de disposer de son propre corps. Et si ce sentiment d'injustice est déjà inscrit dans des références collectives, sa transformation en une conviction politico-morale précise et une action de lutte contre cette injustice

53. Honneth A., La lutte pour la reconnaissance, op. cit., p. 162.

54. Ibid., p. 170.

55. Costalli S. et A. Ruggeri, Symposium: Emotions, Ideologies and Violent Political Mobilization, Cambridge, Juillet 2016. Enzo Nussio, "How ideology channels indeterminate emotions into armed mobilization”, Symposium: Emotions, Ideologies..., op. cit. Wood E., Insurgent Collective Action and Civil War in El Salvador, Cambridge, Cambridge University Press. 2003. Elle y analyse notamment l'impact de l'indignation morale («moral outrage»). Sur le rapport entre ordres normatifs, particulièrement les définitions d'une justice économique, et violence armée en Colombie, voir Grajales J., «Violence Entrepreneurs, Law and Authority in Colombia ", Development and Change, vol. 47, $\mathrm{n}^{\circ}$ 6, 2016, pp. 1294-1315.

56. Viterna J., Women at War: The Micro-Processes of Mobilization in El Salvador, Oxford University Press, 2013. 
relève d'un processus qui fait réapparaitre toute l'importance du travail des organisations politiques. Gamson ajoute ainsi au cadre de l'injustice celui de l'agency : à la dénonciation de l'injustice, les organisations politiques adjoignent l'idée qu'il est possible de lutter contre, la définition d'un ennemi et des moyens d'action ${ }^{57}$. Dans les contextes démocratiques occidentaux contemporains, on l'a vu, l'accent est mis sur le travail de sensibilisation des organisations et de définition de la cause et de l'ennemi, face à des expériences d'injustice diffuses. En contexte coercitif, la référence au sentiment d'injustice s'appuie sur des interpellations émotionnelles fortes, rapportées à la violence, la définition de l'ennemi ou de l'oppresseur étant le plus souvent déjà effective. Si le travail de sensibilisation des organisations est de moindre importance, de même que celui de la cristallisation du sentiment d'injustice autour de figures d'oppression ou de responsabilités, il n'en reste pas moins que le travail de définition de la cause et son inscription précise dans une idéologie restent essentiels. L'enquête menée en Jordanie auprès d'une soixantaine de militants de longue durée islamistes, nationalistes et communistes, montre combien cet apprentissage idéologique s'effectuait, chemin faisant, au cours de l'engagement. Bruno Lefort, dans son étude de la politisation d'étudiants à Beyrouth, observe à nouveau la façon dont les convictions se construisent après l'engagement au sein des organisations ${ }^{58}$. On est loin ici de ce qu'on aurait pu considérer comme des adhésions idéologiques fondées sur un accord politique précis avec telle ou telle organisation. Un militant communiste jordanien souligne, ainsi, qu'il voulait avant tout s'engager, que dans son lycée où les communistes étaient influents il est devenu communiste, dans un autre, il aurait pu devenir islamiste ${ }^{59}$. Certes, la mise en avant de cette indétermination idéologique de départ est aussi une manière de souligner l'authenticité du sentiment d'injustice qui s'exprimait alors indépendamment des organisations et, par là même, l'authenticité que le rapport aux émotions donne à l'engagement. Elle n'enlève rien cependant à ses convictions communistes, qui désormais font corps et vision du monde et peuvent même être retournées contre l'organisation accusée de trahir ses propres idéaux. Le même processus peut s'observer auprès des déçus de l'islamisme, qui retournent contre les Frères musulmans, accusés de corruption, d'opportunisme et de double discours, l'idéologie même de l'organisation, ici trahie ${ }^{60}$.

57. Gamson W., Talking Politics, op. cit.

58. Larzillière P., "Sociologie de l'engagement à partir du Proche-Orient » in Husseini J.et A. Signoles (dir.), op. cit., pp. 179-188 et 299-301. Lefort B., “The Art of Bypassing: Students” Politicization in Beirut", Mediterranean Politics, vol. 22, n 3, 2016, p. 12. Khalila Aude Coëffic souligne aussi cet aspect dans son analyse des dispositifs de sensibilisation du Hezbollah (Coëffic K., Les mobilisations du Hezbollah et la cause palestinienne. Les raisons évolutives des émotions militantes, thèse de doctorat, Institut d'Études Politiques d'Aix-enProvence, 2018). Calabrese Ch., Militer au Hezbollah, Karthala/IFPO, Paris-Beyrouth, 2016.

59. Enquête de terrain en Jordanie, Amman, 2007.

60. Enquête de terrain en Jordanie, Amman, 2009 et 2010. 
Ainsi, s'il est d'usage d'insister sur le travail de pré-formatage des organisations, et en particulier des organisations islamistes, comme le montre à nouveau Carrie R. Wickham pour les Frères musulmans en Égypte ${ }^{61}$, même dans ces contextes de continuité sociale, la construction de la conviction post-adhésion et le façonnage organisationnel sont importants. La référence à l'injustice est reformulée et mise en sens au sein de l'organisation, inscrite dans une vision du monde qui permet aussi au militant de réinterpréter sa propre position. Un processus dont l'importance a été mise au jour dans d'autres contextes, par exemple pour les engagements communistes en France ${ }^{62}$. Lorsqu'il est ainsi énoncé par les militants, le sentiment d'injustice est lié à un rapport à l'action, il ne s'agit pas d'un simple constat de situation injuste, à l'appel à l'indignation partagée, inclus dans l'expression même de ce sentiment d'injustice, s'ajoute le registre de la lutte et de la réparation. Dans cette articulation apparaît tout le travail des organisations de promotion d'un répertoire d'action, de socialisation militante et de mise en sens de la lutte autour de ce répertoire. On peut prendre comme exemple dans les contextes qui nous préoccupent, sans exhaustivité, l'invention d'un répertoire d'action spécifique lors de la première intifada, combinant projet de lutte et projet de société avec manifestations, grèves, boycott et promotion de l'autonomisation alimentaire, un répertoire basé sur des mobilisations de masse. Ou, autre exemple, la circulation en 2011 en Jordanie - suite à de nombreuses mobilisations syndicales antérieures - du répertoire d'action du début des soulèvements arabes, caractérisé par une double appropriation de l'espace public : occupation de la rue et des places, avec sit-in, manifestations, marquage du territoire au moyen d'affiches et de graffitis, etc., et création d'un espace public virtuel à travers un usage extensif d'Internet et des médias sociaux. Si l'analyse des différents répertoires d'action promus dans ce type de contexte dépasserait le cadre de cet article, notons cependant que la notion de répertoire d'action prend en considération l'impact des politiques de répression, des logiques instrumentales, mais aussi des logiques expressives ${ }^{63}$. Il importe d'ajouter à l'analyse de ces répertoires l'enjeu de leur perception, qui ne recoupe pas exactement la question de leur efficacité réelle, une perception en évolution qui joue un rôle dans la modification des formes de l'engagement ${ }^{64}$.

61. Wickham C., Mobilizing Islam. Religion, Activism and Social Change in Egypt, Columbia University Press, 2002, Chapitre VI “'The Call to God'. The Islamist Project of Ideological Outreach", pp. 119-149.

62. Voir Ethuin N., « De l'idéologisation de l'engagement communiste. Fragments d'une enquête sur les écoles du PCF (1970-1990) ", Politix, vol. 16, n 63, troisième trimestre 2003, pp. 145168. Johsua F., « Repenser la production sociale de la révolte. À partir d'une étude des transformations du recrutement à la LCR depuis 2002 ", Revue française de science politique, vol. 63, n 5, 2013, pp. 841-864. Pudal B., « La vocation communiste et ses récits » in Lagroye J. (dir.), La Politisation, Paris, Belin, 2003, pp. 147-161.

63. McAdam D., Tarrow S. et Ch. Tilly, "Pour une cartographie de la politique contestataire ", Politix, n' 41, 1998, p. 16.

64. On peut prendre ici l'exemple de l'évolution de ces perceptions de la première à la deuxième intifada. Larzillière P., "Fermeture ou transformation des "possibles protestataires" ? L'évolution de l'engagement politique dans les Territoires palestiniens », in Benbassa E. (dir.), Israël/Palestine : État des lieux, Paris, CNRS éditions, 2010, pp. 195-207. 
Les énonciations militantes d'un sentiment d'injustice à l'origine d'un engagement s'appuient en contexte coercitif sur la référence à des expériences violentes, des scènes liminaires vues ou vécues (la notion de trauma est d'ailleurs parfois utilisée et son usage dans une sociologie des émotions reste à élaborer de manière plus précise). D'autres types d'expériences sont aussi mobilisés : non plus une scène spécifique, mais l'usure d'une situation où ne cessent de se répéter les humiliations, et les récits transmis de l'injustice par les parents ou les grands-parents. La référence à une violence politique vécue est omniprésente. Les éléments apportés ici invitent à prendre en compte les liens entre type de régime politique et mode d'expression d'un sentiment d'injustice en rapport à l'engagement. La coercition politique se traduit par une violence qui peut être quotidienne, une répression plus ou moins générale, ciblant directement ou indirectement les militants, et crée un contexte où les émotions politiques, les chocs moraux ont une intensité sans commune mesure, et surtout se répètent, sans sortie de la situation. Dans l'analyse de l'engagement dans de tels contextes, il importe de ne pas mettre cet aspect de côté, ne pas l'oublier dans une approche désincarnée et stratégique des militants. En s'appuyant sur les réflexions d'Axel Honneth autour de l'expérience de l'injustice - ou plutôt, dans son vocabulaire, de mépris - on peut rendre compte du caractère particulièrement fondamental de l'expérience de la violence, non seulement du fait de la souffrance infligée, mais en ce qu'elle signifie : de remise en cause de l'intersubjectivité dans les liens sociaux, de destruction de la dignité, et d'aliénation pour un acteur privé de la disposition de son propre corps. De ce fait, les émotions qui sont associées non seulement sont particulièrement intenses, mais s'inscrivent aussi dans le registre spécifique des sentiments moraux.

Pour autant, cette prégnance émotionnelle et ces interpellations existentielles, car il y est bien souvent question aussi de vie ou de mort, ne doivent pas faire oublier toute distanciation et basculer dans une fausse évidence, celle d'un contexte choquant nourrissant un sentiment d'injustice qui conduirait nécessairement à l'engagement. Le récit des expériences violentes et leur inscription dans un cadre d'injustice renvoient également à des mises en cohérence rétrospective, des sélections sont effectuées pour s'inscrire dans des référents partagés. Si une des spécificités du sentiment d'injustice, tel qu'exprimé par les militants, est de toujours s'articuler à une remise en cause et un appel à la lutte, cela renvoie également à un registre de légitimation de la lutte. Or, l'expression du sentiment d'injustice dépasse très largement le cadre militant. Son énonciation implique une dimension morale plus ou moins explicite et invite à dépasser la particularité de telle ou telle expérience, mais un lien express avec une lutte n'est pas toujours effectué. En ce sens, les explications sui generis de l'engagement en référence à un sentiment d'injustice sont à relativiser. Cela reviendrait à un nouveau réductionnisme, non stratégique, mais 
émotionnel cette fois, qui tendrait à faire des émotions une variable unique ; l'usage du sentiment d'injustice pour dénommer autrement une frustration relative peut parfois y renvoyer. On peut ici penser tout à la fois avec et contre Axel Honneth.

Avec Axel Honneth en ce qui concerne sa critique des explications des mouvements sociaux et de l'engagement tournant uniquement autour des intérêts. Une dimension qui est, par exemple, absolutisée avec l'acteur « rationnel » et calculateur des théories de la mobilisation des ressources. Non sans effets parfois paradoxaux, la fameuse analyse de Mancur Olson montre qu'au sein des organisations, même si les différents acteurs ont des intérêts communs, l'accès aux ressources est possible sans participation (free rider) et qu'il faut donc des incitations sélectives positives ou négatives pour pousser l'individu à l'action collective ${ }^{65}$. Ces théories butent cependant sur la question de la définition des intérêts et des bénéfices, définition qui est toujours aussi sociale. Des rétributions économiques aux rétributions symboliques, de la prise en compte des processus d'identification, de désir de reconnaissance ou d'émotions, elles ne cessent de sortir de leur propre cadre pour déterminer les choix des acteurs ${ }^{66}$. Des préférences morales, par exemple, peuvent être intégrées au calcul pour expliquer une coopération qui pourrait paraître opposée à la maximisation de l'intérêt personnel ${ }^{67}$. Pour maintenir l'analyse en termes de coûts-bénéfices y sont inclues des considérations sur les bénéfices symboliques liés à un sens collectif et à des constructions sociales qu'il est, certes, nécessaire de prendre en considération, mais qui ne peuvent être explicitées dans ce cadre théorique. Sans évacuer la question des rétributions du militantisme, il s'agirait alors de prendre aussi en compte dans l'analyse la spécificité des sentiments moraux qui impliquent, par leurs références morales, une inscription dans une intersubjectivité et des représentations axiologiques de ce que devrait être la société (sans nécessairement adhérer à la vision téléologique d'Axel Honneth, qui rapporte la lutte pour la reconnaissance à « un processus historique de progrès moral 68 » dont les divers événements et luttes sont autant d'étapes, vision liée à son adossement partiel aux théorisations hégéliennes).

Contre Axel Honneth pour continuer à interroger, même dans les expériences extrêmes, la façon dont des situations inégalitaires deviennent injustes et inacceptables, et dont les énonciations du sentiment d'injustice associées à l'engagement s'inscrivent dans des cadres et des références liés en partie au tra-

65. Olson M., Logique de l'action collective, Paris, Presses Universitaires de France, 1978 (préf. de Raymond Boudon, trad. de Mario Levi).

66. Voir notamment l'analyse de Cefaï D., Pourquoi se mobilise-t-on? Les théories de l'action collective, Paris, La Découverte, 2007, pp. 214-215.

67. Lopes H., «Utilité, normes et sentiments moraux. De leur incommensurabilité », Revue $d u$ MAUSS, vol. 25, n 1, 2005, pp. 404-427.

68. Honneth A., La lutte pour la reconnaissance, op. cit., p. 201. 
vail des organisations : mise en sens de l'injustice et promotion des répertoires d'action spécifiques, dont une des fonctions est la réouverture d'horizons d'attente, malgré la répression. 